\title{
Estilos parentais, bullying e o papel mediador da sintomatologia psicopatológica em adolescentes e jovens adultos
}

\author{
Catarina Pinheiro Mota1,2 \& Mónica Pinheiro ${ }^{1}$ \\ 1 Universidade de Trás-os-Montes e Alto Douro - UTAD \\ 2 Centro de Psicologia da Universidade do Porto
}

\begin{abstract}
Resumo: 0 bullying constitui um fenómeno que embora tenha vindo a assumir grandes repercussões nas últimas décadas, continua a carecer de uma abordagem compreensiva e relacional face às vivências dos jovens. 0 presente estudo teve como objetivo analisar os estilos parentais percebidos, quer relativamente à mãe, quer relativamente ao pai, e testar o seu papel no envolvimento em comportamentos de bullying, assim como, analisar o papel mediador da sintomatologia psicopatológica na associação anterior. A amostra foi constituída por 1976 adolescentes e jovens adultos, com idades compreendidas entre os 14 e os 25 anos de idade $(M=17.22 ; D P=2.50)$. Os resultados apontam para uma associação significativa dos estilos parentais dos pais face aos comportamentos de bullying e ainda para existência de um papel mediador da sintomatologia psicopatológica na associação entre estilos parentais e comportamentos de bullying. Os resultados serão discutidos à luz da teoria de Baumrind, assumindo a importância dos estilos parentais para o desenvolvimento psicológico e comportamental nos adolescentes e jovens adultos.
\end{abstract}

Palavras-chave: Estilos parentais; bullying; sintomatologia psicopatológica; adolescentes e jovens adultos.

Parental styles, bullying and the mediational role of psychopathological symptoms in adolescents and young adults: Over the last decades, the bullying phenomenon had countless repercussions, however, it still remains an issue that need to be addressed according to a comprehensive and relational approach of young's experiences. The present study aims to analyse the perceived parenting styles (either to mother or the father) and to test their involvement on bullying behaviours, as well as to test the mediating role of the psychopathological symptomatology in last association. The study sample consisted of 1976 adolescents and young adults aged between 14 and 25 years old $(\mathrm{M}=17.22, \mathrm{SD}=2.50)$. The results indicate a significant effect of parenting styles regarding to bullying behaviours, as well as the existence of a mediation role of psychopathological symptomatology in the association between parenting styles and bullying behaviours. The results will be discussed in light of Baumrind's theory, assuming the importance of parenting styles to the psychological and behavioural development in adolescents and young adults.

Keywords: Parental styles; bullying; psychopathological symptomatology; adolescents and young adults.

Papel dos estilos parentais no desenvolvimento de adolescentes e jovens adultos

De acordo com a teoria da vinculação preconizada por Bowlby (1969), a vinculação é designada pela criação de laços de afetividade com as figuras significativas. Estes laços de afetividade propiciam a segurança e proteção da criança aquando de situaç̃ões de risco, capacitando a criança para a exploração de si, dos outros e do meio envolvente. Bowlby (1969) aponta a existência de ligações seguras e inseguras, podendo estas predizer a qualidade das relações que o indivíduo desenvolve ao longo da sua vida.

Bowlby (1980) sugere que as diferenças na qualidade da interação nas relações construídas durante a infância têm um papel na construção dos modelos internos dinâmicos ao longo da vida. Assim, os estilos parentais assumem um papel de destaque, pois a dinâmica e a natureza das relações estão relacionadas com o ambiente parental (Michiels, Grietens, Onghena, \& Kuppens, 2010). As figuras cuidadoras possuem uma relevância no que concerne ao impacto que têm no desenvolvimento cognitivo, emocional e relacional dos filhos (Bowlby, 1973). A qualidade dos estilos parentais implementados na infância, pode contribuir para o desenvolvimento mental futuro, uma vez que a construção das primeiras representações surge na interação com as figuras cuidadoras (Nishikawa, Sundbom, \& Hägglöf, 2010). Os

\footnotetext{
${ }^{1}$ Morada para correspondência: Catarina Pinheiro Mota, Universidade de Trás-os-Montes e Alto Douro (UTAD). Departamento de Educação e Psicologia - Edifício da Escola de Ciências Humanas e Sociais - Polo I, Quinta dos Prados - UTAD, 5000-801 Vila Real, Portugal. E-mail: catppmota@utad.pt. Esta investigação foi parcialmente financiada pela FCT através do projeto PEst-C/PSI/UI0050/2011 e os fundos FEDER através do programa COMPETE com o projeto FCOMP-01-0124-FEDER-022714.
} 
estilos parentais caracterizam a natureza afetiva e a relação entre os pais e filhos, apresentando por isso, uma contribuição importante para o desenvolvimento psicossocial do adolescente e jovem adulto. 0 estilo parental é definido pelas dimensões: controlo, maturidade, comunicação e disponibilidade afetiva parental com os filhos, sugerindo um papel importante na construção de alguns comportamentos que os adolescentes possam apresentar na relação com o exterior, nomeadamente com os pares e mais tarde nas demais relações enquanto jovens adultos (Baumrind, 1991,1997).

Baumrind $(1966,1991)$ propôs uma abordagem tipológica onde agrega os estilos parentais tendo por base o controlo parental no comportamento do adolescente, com o estilo democrático ao centro, considerado pelo autor como o mais equilibrado, e os extremos, o estilo autoritário, e o permissivo. De acordo com a autora, o estilo democrático é caracterizado pela presença de limites, regras e independência; é um estilo onde existe controlo, mas com moderação, onde o ambiente familiar é caloroso, estimulante e existe comunicação clara e aberta baseada no respeito mútuo. Em contraste, o estilo autoritário é pautado pela exigência, obediência, autoridade, baixo nível de afetividade e comunicação, onde os comportamentos e ações dos filhos são avaliados de forma rígida e inflexível, sendo comum a utilização de castigos e punições. No extremo oposto encontra-se o estilo permissivo, caracterizado pela tolerância excessiva e baixo nível de exigência, indulgência e ausência de regras ou punições, com a ausência de controlo ou autoridade, os indivíduos são livres de tomarem as próprias decisões, mas com a ausência de uma base de suporte que os oriente quando necessário (Baumrind, 1991).

Alguma literatura indica que adolescentes e jovens adultos que vivenciam o seio de um estilo democrático detêm maiores capacidades de ultrapassar vivências e sentimentos negativos (e.g. Morris, Silk, Steinberg, Myers, \& Robinson, 2007; Silva, Morgado, \& Maroco, 2012), contrastando com filhos que vivem num estilo autoritário ou permissivo, que se apresentam por vezes como sendo mais vulneráveis no desenvolvimento de certas sintomatologias psicopatológicas, como a ansiedade ou depressão (e.g. Hutz \& Bargagir, 2006). Esta vulnerabilidade é particularmente visível em ambientes familiares com carência de afeto e vínculos inseguros com as figuras significativas de afeto (Simões, Ferreira, Braga, \& Vicente 2015). Assim, sugere-se que os estilos parentais assumam relevância na compreensão das condutas ao longo do desenvolvimento do adolescente e jovem adulto. 0 desenvolvimento do adolescente decorre num período de muitas alterações, quer a nível psicológico, biológico e social que podem conduzir a instabilidade psíquica, mas também sugerem ser uma importante oportunidade para a formação de uma identidade (Erikson, 1968; Macedo, 2010). 0 processo de separação individuação adaptativo constitui um processo evolutivo que ocorre na díade relacional entre pais e filhos, estende-se até à idade adulta e promove o crescimento pessoal e sentido de autonomia dos jovens (e.g. Arnett, 2006; Bynner, 2005; Mota \& Rocha, 2012). Scabini e Manzi (2011) acrescentam que um contexto familiar favorável, com relacionamentos de qualidade, propicia um processo de individuação bem sucedido aos adolescentes e jovens adultos.

\section{0 papel dos estilos parentais no desenvolvimento de sintomatologia psicopatológica e comportamentos de bullying}

O estilo parental adotado pelas figuras cuidadoras pode apresentar um papel preditor na saúde mental dos adolescentes e jovens adultos. Os estilos parentais parecem associar-se a fatores latos que englobam a forma de relacionamento abrangente com os filhos, na medida em que a consistência do tipo de disciplina, o nível de envolvimento, o acompanhamento, a presença e apoio no dia a dia e o tipo de comunicação, são fatores de grande importância no que diz respeito à saúde mental dos jovens (Newman, Harrison, Dashiff, \& Davies, 2008; Skinner, Jonhson, \& Snyder, 2005). 0 estilo parental democrático parece associar-se a menos sintomas depressivos e ansiosos por parte do jovem (e.g. Hutz \& Bardagir, 2006; Laboviti, 2015; Lipps et al., 2012). Por outro lado, Olweus (1991) verificou que num estilo parental mais autoritário ou permissivo parecem predominar comportamentos mais agressivos com os pares. Silva, Morgado e Maroco (2012), corroboram esta predominância ao identificar que os jovens educados neste meio parecem ser os que mais se encontram envolvidos em comportamentos de bullying. A educação praticada pelos pais na relação com os filhos tem um papel relevante na forma como estes vão interagir com os pares.

As condições familiares a que os adolescentes e jovens adultos estão expostos possuem um papel na construção de pensamentos e aquisições de comportamentos que podem ter um efeito na interação social (Dodge \& Petit, 2003; Pontzer, 2010). A literatura suporta ainda alguma relação dos estilos parentais autoritário e permissivo com o aumento dos níveis de ansiedade e depressão (e.g. Reppold \& Hutz 2003; Wolfradt, Hempel, \& Miles, 2003). Esta associação poderá conduzir ao desenvolvimento de comportamentos menos adaptativos que podem oscilar entre a introversão e extroversão dos jovens, onde se inclui o bullying. Os estilos parentais parecem apresentar uma relação com a sintomatologia 
psicopatológica e na promoção de violência e comportamentos agressivos, sendo possível referir que estes estilos parentais praticados poderão predizer a existência de comportamentos de bullying (Atik \& Güneri, 2013). Esta relação corroborada por vários autores, sendo o estilo parental autoritário e o permissivo associados a comportamentos de vitimização e agressão (e.g. Georgiou, 2008; Powell \& Ladd, 2010), enquanto o estilo democrático é frequentemente associado ao não envolvimento em comportamentos agressivos (e.g. Hokoda, Lu, \& Angeles, 2006). O bullying é caracterizado por um conjunto de comportamentos negativos, como agressão física ou psicológica, que um indivíduo ou um grupo realiza de forma repetida e ao longo do tempo a outro indivíduo ou grupo, podendo estes comportamentos ter origem em diferenças sociais, étnicas, religiosas, género, orientação sexual, aparência, desequilíbrios de forças, entre muitas outras (Olweus \& Limber, 1999). Por vezes, estes comportamentos são desvalorizados ou ignorados, tanto por parte dos pais como dos professores, sendo vistos como comportamentos normais, próprios do crescimento (Dracic, 2009; Neto, 2005; Skrzypiec, Slee, Murray-Harvey, \& Pereira, 2011). Algumas situações de participação em comportamentos de bullying, como vítima ou agressor, advêm de alterações psicológicas que podem persistir nas pessoas que o vivenciaram durante a adolescência e jovem adultícia (Carvalhosa, 2010).

Hokoda et al. (2006), num estudo realizado com 325 participantes com idades entre os 13 e 16 anos, e os seus respetivos pais verificou que os adolescentes que possuíam uma relação mais positiva com os seus pais, pautada pela independência, apesar da existência de algumas regras e limites, apresentavam menos probabilidade de se encontrarem envolvidos em comportamentos de bullying. Estes investigadores verificaram ainda que os jovens educados em estilos democráticos desenvolviam mais comportamentos pró-sociais com os seus pares do que os educados em estilos autoritários. Estes resultados são consistentes com uma grande parte da literatura desta temática, destacando o benefício do estilo democrático para bem-estar do jovem (e.g. Georgiou, 2008). Na mesma linha, Powell e Ladd (2010) sugerem ainda que pais considerados mais conflituosos e numa relação menos coesiva tendem a afetar o comportamento futuro dos filhos. Esta questão coloca-se na medida em que, segundo os autores, estas famílias são normalmente caracterizadas como apresentando uma maior distância emocional entre os membros e normalmente com presença de punição física por parte dos pais.

As vítimas de bullying tendem a caracterizar os seus pais como sendo demasiado protetores, inversamente, os agressores aparecem mais frequentemente em famílias que punem e castigam demasiado os seus filhos, geralmente no seio de um estilo autoritário (Cook, Williams, Guerra, Kim, \& Sadek, 2010; Efobi \& Nwokolo, 2014; Georgiou, 2008). Um estudo realizado por Klomek, Marrocco, Kleinman, Schonfeld e Gould (2007), com 2342 participantes, com idades entre os 13 até aos 19 anos, concluiu que não só as vítimas como os agressores de bullying apresentam maiores níveis de depressão e ideação suicida, e que esta se torna ainda mais alta com o aumento de situações de bullying vivenciadas. Ainda, segundo Albino e Terêncio (2012) e Dracic (2009) o bullying, exercido de forma contínua nas vítimas, pode provocar o desenvolvimento de baixa autoestima, stresse, ansiedade, baixo rendimento escolar e surgimento de sintomatologia depressiva e ansiosa, potenciando o desenvolvimento de comportamentos igualmente desadaptativos com os pares. Pelo exposto, sugere-se que o estilo parental adotado pelas figuras primordiais é de grande relevância para o bem-estar físico e psíquico do adolescente e jovem adulto. Torna-se pertinente aprofundar esta temática de investigação, e assim contribuir para a diminuição das repercussões negativas que estas podem ter no futuro dos adolescentes e jovens adultos.

\section{OBJETIVOS E HIPÓTESES}

0 presente estudo tem como principal objetivo analisar em que medida os estilos parentais dos adolescentes e jovens adultos se associam ao comportamento de bullying. Pretende-se ainda testar o efeito mediador da sintomatologia psicopatológica na associação anterior. Quanto aos objetivos específicos, pretende-se analisar em que medida os estilos parentais e sintomatologia psicopatológica variam em função dos comportamentos de bullying. Por último, procura-se testar em que medida a sintomatologia psicopatológica pode exercer um efeito mediador na associação entre os estilos parentais e desenvolvimento de comportamentos de bullying. Tendo em conta os objetivos propostos espera-se que existam diferenças significativas entre os estilos parentais e sintomatologia psicopatológica, relativamente aos comportamentos de bullying. Nesta medida, espera-se que os sujeitos caracterizados com tipologia vítima e "não envolvidos" apresentem maior predominância no estilo democrático e menor predominância no estilo autoritário e permissivo, quando comparados com os sujeitos de tipologia vítima \& agressores e agressores. Por outro lado, espera-se que os sujeitos caracterizados com tipologia vítima e vítima \& agressor apresentem maiores níveis de ansiedade, depressão e sensibilidade quando comparados com os sujeitos com tipologia de agressores e "não envolvidos". Prevê-se, ainda, que a 
sintomatologia psicopatológica exerça um efeito mediador na associação entre os estilos parentais no desenvolvimento de comportamentos de bullying.

\section{MÉTODO}

\section{Participantes}

A amostra deste estudo foi constituída por 1976 indivíduos. Foram selecionadas 12 escolas secundárias da zona norte e a Universidade de Trás-os-Montes e Alto Douro, onde foi possível uma amostra constituída por 714 indivíduos do sexo masculino (36.1\%) e 1262 do sexo feminino (63.9\%), com idades compreendidas entre os 14 e os 25 anos de idade $(M=17.22 ; D P=2.50)$. Quanto às idades das figuras parentais dos participantes, estas variam entre 32 e 75 anos $(M=47.74 ; D P=5.80)$ no pai e entre 30 e 65 anos $(M=45.29 ; D P=5.57)$ na mãe.

\section{Instrumentos}

0 primeiro instrumento do protocolo consistiu num questionário sociodemográfico que abrange as vertentes sociodemográficas, onde foi possível obter informações como idade e sexo.

O Questionário de Exclusão Social e Violência Escolar (QESVE), aferido para a população portuguesa por Martins (2005), trata-se da adaptação do Cuestionario de Evaluacion de la violência entre iguales en la Escuela y en el Ocio (CEVEO) de Diaz-Aguado, Arias e Seoane (2004), onde é avaliado o tipo e o grau de envolvimentos dos indivíduos em comportamentos de bullying. Este questionário é composto por 45 itens divididos em três grupos de resposta, cada um deles com 15 itens, sendo que cada grupo apresenta uma amplitude entre 15 a 60 pontos. Estes grupos representam as seguintes subescalas: Vitimização (15 itens), procurando avaliar o modo como o adolescente se sente em diversas situações com os seus pares ("Os meus colegas insultam-me e gozam-me"); Agressão (15 itens), que permite avaliar a existência de comportamentos agressivos para com os pares ("Rejeitei um(a) colega"); Observação (15 itens), que permite avaliar se o indivíduo assistiu a comportamentos de bullying praticados por outros ("Chamarem nomes que ofendem e ridicularizam um(a) colega"). Os itens estão apresentados numa escala tipo Likert entre 1 ("Nunca") e 4 ("Quase sempre"). A análise da consistência interna demonstrou valores de alfa de Cronbach de .89 para a totalidade do instrumento. No que refere aos alfas de Cronbach de cada dimensão, registam-se valores de .75 para a vitimização, .70 para a agressão e .89 para a dimensão observação. A análise confirmatória apresenta valores ajustados para o modelo, $\chi^{2}(18)=81.77$, $\chi^{2} / g l=4.54, p<.001$, com RMR $=.00$, RMSEA $=.04, \mathrm{CFI}=.99, \mathrm{GFI}=.99, \mathrm{AGFI}=.98$, encontrando-se estes dentro dos valores de referência em que os índices de ajustamento Goodness of Fit Index (GFI) e o Adjustment Goodness of Fit Index (AGFI) e Comparative Fit Index (CFI), se situam acima de .90, assim como os índices de Root Mean Square Residuals (RMR) e Root Mean Square Error of Approximation (RMSEA) apresentam valores abaixo de .08 (Byrne, 1998; Fan, Thompson, \& Wang, 1999; Marôco, 2014a).

Martins e Castro (2010) propuseram uma organização em quatro grupos que permitem identificar o tipo de indivíduos (vítima, agressor, vítima \& agressor e não envolvido) baseando-se na soma das respostas às subescalas vitimização e agressão. 0 agrupamento é realizado com base nas seguintes regras: vítima, na subescala agressão <= 18 e na subescala vitimização >18; agressor, na subescala agressão >18 e na subescala vitimização <=18; vítima \& agressor, na subescala agressão >18 e na subescala vitimização > 18, e não envolvido, na subescala agressor <= 18 e na subescala vitimização <= 18 (Martins \& Castro, 2010). Esta categorização será utilizada para definir os pontos de corte das análises deste trabalho.

O Parental Styles \& Dimensions Questionnaire: Short Version (PSDQ) foi adaptada para a população portuguesa por Nunes e Mota (2018) a partir da versão da versão de Robinson, Mandleco, Olson e Hart (1995), tendo como objetivo avaliar a perceção que os adolescentes têm dos estilos parentais das figuras cuidadoras. Trata-se de um questionário, composto por 32 itens, onde existe a versão pai e a versão mãe. Este questionário divide-se em três estilos parentais: Democrático (15 itens), Autoritário (12 itens); Permissivo (5 itens). Cada estilo parental distribui as dimensões usadas no presente estudo, a saber: Democrático (Apoio e afeto, Regulação e Cedência de Autonomia/Participação Democrática); Autoritário (Coerção física e Punição); e Permissivo (Indulgência). Os itens estão apresentados numa escala tipo Likert entre 1 ("Nunca") e 5 ("Sempre"). A análise da consistência interna demonstrou valores de alfa de Cronbach de .89/.85 para o pai e mãe, respetivamente, para a totalidade do instrumento. No que refere aos alfas de Cronbach de cada subescala registam-se valores de .88/.83 para Apoio e afeto, .87/.83 para Regulação, .86/.82 para a Cedência de Autonomia/Participação Democrática, .74/.74 para a Coerção física, .62/.62 para a Punição e .58/.57 para a Indulgência. A análise confirmatória apresenta índices de ajustados bons para o modelo, $\chi^{2}(116)=898.84, \chi^{2} / g l=7.82, p<.001 / \chi^{2}(116)=$ $782.42, \chi^{2} / g l=6.75, p<.001$, com RMR $=.08 / .07$, RMSEA $=.06 / .05$, CFI $=.96 / .95$, GFI $=.95 / .96$, AGFI $=$ 
.93/.94, encontrando-se estes dentro dos valores de referência em que os índices de ajustamento GFI, AGFI e CFI se situam acima de .90, assim como os índices de RMR e RMSEA apresentam valores abaixo de .08 (Byrne, 2006; Fan et al., 1999; Marôco, 2014a).

O Brief Symptom Inventory (BSI) foi adaptado para a população portuguesa por Canavarro (1999) a partir da versão desenvolvida inicialmente por Derogatis (1993), tendo como objetivo avaliar indicadores de sintomatologia psicopatológica. Trata-se de um instrumento constituído por 53 itens. 0 questionário avalia 9 dimensões, no entanto, no presente estudo apenas objetiva a análise de 3 dimensões: Depressão (6 itens) que coloca questões do tipo "Sentir que não tem valor", Ansiedade (6 itens) que coloca questões como "Nervosismo e tensão interior" e Sensibilidade Interpessoal (4 itens), que coloca questões do tipo "Sentir que as outras pessoas não são suas amigas ou não gostam de si". Os itens são apresentados numa escala tipo Likert que varia entre 0 ("Nunca") e 4 ("Muitíssimas vezes"). Relativamente à análise de consistência interna, demonstrou valor de alfa de Cronbach de .92 relativamente à totalidade das 3 dimensões. No que respeita à consistência interna de cada dimensão, registaram-se valores de alfa de Cronbach de .84 para Depressão, .82 para a Ansiedade e .82 para a Sensibilidade Interpessoal. As análises fatoriais confirmatórias apresentam valores de ajustamento adequados para o modelo $\chi^{2}(19)=73.25, \chi^{2} / g l=3.86, p<.001$, com RMR $=.01$, RMSEA $=.03$, CFI $=1.00$, $\mathrm{GFI}=.99, \mathrm{AGFI}=.98, \mathrm{IFI}=1.00, \mathrm{NFI}=.99$, encontrando-se estes dentro dos valores de referência em que os índices de ajustamento GFI, AGFI e CFI se situam acima de .90, assim como os índices de RMR e RMSEA apresentam valores abaixo de .08 (Byrne, 2006; Fan et al. 1999; Marôco, 2014a).

\section{Procedimentos e estratégia de análise de dados}

A recolha da amostra abrange adolescentes e jovens adultos do 9.. ano de escolaridade até ao ensino superior realizada de forma aleatória em instituições da zona norte do país. A recolha de dados foi realizada durante os meses de outubro, novembro e dezembro de 2015. É de salientar que inicialmente foi realizado um pedido de apreciação e parecer à Direção Geral de Educação e, posteriormente, uma reunião com os Diretores do Conselho Executivo das escolas secundárias, onde foi possível elucidar sobre os objetivos do estudo, explicando a sua pertinência, de forma a obter as devidas autorizações. Procedeuse à entrega do Termo Consentimento Livre e Esclarecido aos encarregados de educação dos jovens a frequentar o ensino secundário. No ensino universitário, a amostra foi recolhida na universidade de acolhimento do projeto situada no interior norte de Portugal, onde foi submetido ao conselho de ética um pedido de apreciação e parecer, solicitando-se também a autorização aos diretores de escolas para administração do questionário. Após a obtenção de parecer positivo por parte das escolas secundárias e da universidade de acolhimento do projeto, foi realizada a recolha de dados.

A presente investigação apresenta um desenho transversal, dado que o conjunto de medições foi realizado num único momento do tempo, não existindo um seguimento temporal dos indivíduos. 0 tratamento dos resultados foi realizado com os programas IBM SPSS® - Statistical Package For Social Sciences e IBM SPSS Amos, ambos na versão 23 para o sistema Microsoft® Windows® 10. Inicialmente foram recolhidos 2000 participantes, não havendo recusas. Procedeu-se posteriormente à limpeza da amostra, onde foram excluídos 5 missings e 19 outliers, devido a ausências de preenchimento de mais de $10 \%$ em alguns questionários, assim como preenchimentos ambíguos e incoerentes (Field, 2005). Foi ainda confirmada a normalidade da amostra (Marôco, 2014b) e procedeu-se a análises estatísticas mediante testes paramétricos.

Para a concretização dos objetivos propostos na presente investigação, realizou-se um conjunto de análises estatísticas na qual se consideraram valores de significância $p<.05$ na interpretação dos dados. Utilizaram-se análises correlacionais, análises de variância (ANOVA) e análises de variância multivariada (MANOVAs). Testou-se ainda a presença de um efeito mediador da variável sintomatologia psicopatológica na associação entre as variáveis estilos parentais e comportamento de bullying. Recorreuse a modelos de equações estruturais com recurso ao programa AMOS, através do método de bootstraping (Maroco, 2014a). Analisou-se o contributo da variável mediadora, sintomatologia psicopatológica, na relação entre a variável dependente e independente.

\section{RESULTADOS}

\section{Variância dos estilos parentais do pai em função de tipologias de comportamento de bullying}

No sentido de realizar a análise de variância dos estilos parentais, procedeu-se à criação de tipologias de comportamento de bullying tendo em conta o seu valor de corte pré-estabelecido pelo autor (Martins, 2009). A agregação dos indivíduos foi distribuída em 4 clusters, a saber: vítima $(n=238)$, agressor $(n=$ $154)$, vítima \& agressor $(n=185)$ e não envolvidos $(n=1399)$. 
De seguida, procedeu-se à realização de análises multivariadas (MANOVAs) com o intuito de analisar as diferenças entre as variáveis estilos parentais do pai e da mãe face à tipologia de comportamento de bullying. As comparações múltiplas entre os grupos foram realizadas a partir de testes post-hoc tendo-se utilizado o teste de Scheffé.

Relativamente às diferenças dos estilos parentais do pai face à tipologia de comportamento de bullying, os resultados apontam para a existência de diferenças significativas $F(9,5916)=8.42 ; p=.001$; $\eta^{2}=1.0$, verificando-se assim diferenças na variável pai democrático, $F(3,1972)=6.49 ; p=.001 ; \eta^{2}=$ .971. Os indivíduos vítima \& agressores $(M=3.29: D P=.87$, com IC 95\% $[3.15,3.43])$ denotam um estilo democrático no pai menor do que os indivíduos não envolvidos $(M=3.59 ; D P=.96$, com IC 95\% [3.53, 3.64]), não se tendo constatado diferenças entre o estilo parental democrático nas vítimas e agressores. Registam-se também diferenças significativas na variável pai autoritário $F(3,1972)=14.43 ; p=.001 ; \eta^{2}=$ 1.0 , mostrando, que os indivíduos agressores $(M=2.07 ; D P=.51$, com IC 95\% $[1.99,2.15])$ e o tipo vítima $\&$ agressor $(M=2.07 ; D P=.50$, com IC 95\% $[1.99,2.14])$ denotam um estilo autoritário no pai maior do que os indivíduos vítima $(M=1.99 ; D P=.52$, com IC 95\% $[1.92,2.05])$ e estes indicam um estilo autoritário no pai maior do que os indivíduos não envolvidos $(M=1.88$; $D P=.50$, com IC 95\% [1.85, 1.90]). Registam-se, também, diferenças significativas no estilo parental permissivo do pai $F(3,1972)=$ $6.73 ; p=.001 ; \eta^{2}=.976$. As comparações post-hoc indicam que indivíduos agressores $(M=2.09 ; D P=.78$, com IC 95\% [1.98, 2.19]) denotam no pai um estilo parental mais permissivo, comparativamente aos indivíduos vítima \& agressor $(M=2.08 ; D P=.69$, com IC 95\% [1.98, 2.17]) e aos indivíduos não envolvidos $(M=1.90 ; D P=.68$, com IC 95\% [1.86, 1.93]), (Tabela 1).

Tabela 1. Análise diferencial dos estilos parentais do pai face à tipologia do bullying

\begin{tabular}{|c|c|c|c|c|}
\hline Estilos parentais & Tipologia de Bullying & $\mathbf{n}$ & $M(\mathrm{DP})$ & $\begin{array}{c}\text { Direção das diferenças significativas } \\
\text { (Teste Scheffé) }\end{array}$ \\
\hline \multirow{4}{*}{ Democrático } & 1 - Vítima & 238 & $3.44(1.00)$ & \multirow{4}{*}{$3<4$} \\
\hline & 2 - Agressor & 154 & $3.48(0.90)$ & \\
\hline & 3 - Vítima \& Agressor & 185 & $3.29(0.87)$ & \\
\hline & 4 - Não envolvido & 1399 & $3.59(0.96)$ & \\
\hline \multirow{4}{*}{ Autoritário } & 1 - Vítima & 238 & $1.99(0.52)$ & \multirow{4}{*}{$\begin{array}{c}1>4 \\
2>4 \\
3>4 \\
2 \& 3>1\end{array}$} \\
\hline & 2 - Agressor & 154 & $2.07(0.51)$ & \\
\hline & 3 - Vítima \& Agressor & 185 & $2.07(0.50)$ & \\
\hline & 4 - Não envolvido & 1399 & $1.88(0.50)$ & \\
\hline \multirow{4}{*}{ Permissivo } & 1 - Vítima & 238 & $1.96(0.66)$ & \multirow{4}{*}{$\begin{array}{l}2>4 \\
3>4\end{array}$} \\
\hline & 2 - Agressor & 154 & $2.09(0.78)$ & \\
\hline & 3 - Vítima \& Agressor & 185 & $2.08(0.69)$ & \\
\hline & 4 - Não envolvido & 1399 & $1.90(0.68)$ & \\
\hline
\end{tabular}

\section{Variância dos estilos parentais da mãe em função de tipologias de comportamento de bullying}

No que concerne às diferenças do estilo parental na mãe face à tipologia de comportamento de bullying, os resultados apontam para a presença de diferenças significativas $F(9,5916)=7.07 ; p=.001 ; \eta^{2}=1.0$, verificando-se diferenças na variável mãe democrática $F(3,1972)=6.02 ; p=.001 ; \eta^{2}=.959$. Tendo em conta comparações post-hoc, os indivíduos vítima \& agressores $(M=3.64: D P=.76$, com IC 95\% [3.52, 3.76]) denotam um estilo democrático na mãe maior do que os indivíduos não envolvidos $(M=3.26 ; D P=$ .80, com IC 95\% [3.82 3.90]), não se tendo constatado diferenças entre o estilo parental democrático nas vítimas e agressores. Verificam-se diferenças significativas na variável mãe autoritária $F(3,1972)=$ 12.44; $p=.001 ; \eta^{2}=1.00$, mostrando assim, através das comparações post-hoc que os indivíduos agressores $(M=2.13 ; D P=.47$, com IC 95\% $[2.05,2.09])$ e vítima \& agressor $(M=2.15 ; D P=.56$, com IC $95 \%[2.07,2.22])$ denotam um estilo autoritário na mãe maior do que os indivíduos vítima $(M=2.07 ; D P$ $=.53$, com IC 95\% $[2.01,2.13])$ e estes indicam um estilo autoritário na mãe maior do que os indivíduos não envolvidos $(M=1.96 ; D P=.50$, com IC 95\% [1.94, 1.99]). Registam-se, também, diferenças significativas no estilo parental permissivo na mãe $F(3,1972)=9.14 ; p=.001 ; \eta^{2}=.996$. As comparações post-hoc indicam que indivíduos agressores $(M=2.23 ; D P=.77$, com IC 95\% $[2.03,2.20])$ denotam na mãe um estilo parental mais permissivo, comparativamente aos indivíduos vítima \& agressor $(M=2.19$; $D P=.73$, com IC 95\% $[2.09,2.29])$ e aos indivíduos não envolvidos $(M=2.00 ; D P=.69$, com IC 95\% [1.96, 2.03]) (Tabela 2). 
Tabela 2. Análise diferencial dos estilos parentais da mãe face à tipologia do bullying

\begin{tabular}{llccc}
\hline Estilos parentais & Tipologia de Bullying & $\mathbf{n}$ & $\boldsymbol{M}(\mathbf{D P})$ & $\begin{array}{c}\text { Direção das diferenças significativas } \\
\text { (Teste Scheffé) }\end{array}$ \\
\hline \multirow{2}{*}{ Democrático } & 1 - Vítima & 238 & $3.77(0.84)$ & $3<4$ \\
& 2 - Agressor & 154 & $3.70(0.72)$ & \\
& 3 - Vítima \& Agressor & 185 & $3.64(0.76)$ & $1>4 ;$ \\
& 4 - Não envolvido & 1399 & $3.86(0.80)$ & $3>4 ;$ \\
Autoritário & 1 - Vítima & 238 & $2.07(0.53)$ & \\
& 2 - Agressor & 154 & $2.13(0.47)$ & $2>4 ;$ \\
& 3 - Vítima \& Agressor & 185 & $2.15(0.56)$ & $3>4$ \\
\hline
\end{tabular}

Variância da sintomatologia psicopatológica em função das tipologias de comportamento de bullying

No que concerne às diferenças da sintomatologia psicopatológica face à tipologia de comportamento de bullying, os resultados apontam para a presença de diferenças significativas $F(9,5916)=25.35 ; p=.001$; $\eta^{2}=1.00$, verificando-se diferenças na variável ansiedade $F(3,1972)=39.04 ; p=.001 ; \eta^{2}=1.00$. Tendo em conta comparações post-hoc, de acordo com o teste de Scheffé os indivíduos vítima $(M=2.16 ; D P=.73$, com IC 95\% [2.07, 2.24]) e vítima \& agressor $(M=2.13$; $D P=.70$, com IC 95\% [2.04, 2.23]) apresentam maior ansiedade comparativamente aos indivíduos agressor $(M=1.82$; $D P=.57$, com IC 95\% [1.72, 1.92]) e aos não envolvidos $(M=1.76 ; D P=.63$, com IC 95\% [1.72, 1.79]). Registam-se, também, diferenças significativas na depressão $F(3,1972)=65.11 ; p=.001 ; \eta^{2}=1.00$. Atendendo às comparações post-hoc, elas indicam que os indivíduos vítima \& agressor $(M=2.18$; $D P=.76$, com IC 95\% [2.09, 2.28]) e vítima (M $=2.16 ; D P=.83$, com IC 95\% $[2.08,2.25])$ apresentam maior depressão comparativamente aos indivíduos agressor $(M=1.74 ; D P=.54$, com IC 95\% [1.64, 1.84]) e aos não envolvidos $(M=1.66 ; D P=.62$, com IC $95 \%[1.62,1.69])$. São igualmente observadas diferenças significativas na variável sensibilidade interpessoal $F(3,1972)=78.35 ; p=.001 ; \eta^{2}=1.00$. As comparações post-hoc indicam que os indivíduos vítima \& agressor $(M=2.24 ; D P=.82$, com IC 95\% $[2.14,2.35])$ e vítima $(M=2.24 ; D P=.87$, com IC 95\% $[2.14,2.33])$ apresentam maior sensibilidade interpessoal quando comparados com aos indivíduos agressor $(M=1.72 ; D P=.61$, com IC $95 \%[1.60,1.83])$ e aos não envolvidos $(M=1.63 ; D P=.69$, com IC 95\% [1.59, 1.67]) (Tabela 3).

Tabela 3. Análise diferencial da sintomatologia psicopatológica face à tipologia do bullying

\begin{tabular}{|c|c|c|c|c|}
\hline $\begin{array}{l}\text { Sintomatologia } \\
\text { Psicopatológica } \\
\end{array}$ & Tipologia de Bullying & $\mathbf{n}$ & $M$ (DP) & $\begin{array}{c}\text { Direção das diferenças significativas } \\
\text { (Teste Scheffé) }\end{array}$ \\
\hline \multirow{4}{*}{ Ansiedade } & 1 - Vítima & 238 & $2.16(0.73)$ & \multirow{4}{*}{$\begin{array}{l}1>2 \\
1>4 \\
3>2 \\
3>4\end{array}$} \\
\hline & 2 - Agressor & 154 & $1.82(0.57)$ & \\
\hline & 3 - Vítima \& Agressor & 185 & $2.13(0.70)$ & \\
\hline & 4 - Não envolvido & 1399 & $1.76(0.63)$ & \\
\hline \multirow{4}{*}{ Depressão } & 1 - Vítima & 238 & $2.16(0.83)$ & \multirow{4}{*}{$\begin{array}{c}1>2 \\
1>4 \\
3>2 \\
3>4\end{array}$} \\
\hline & 2 - Agressor & 154 & $1.74(0.54)$ & \\
\hline & 3 - Vítima \& Agressor & 185 & $2.18(0.76)$ & \\
\hline & 4 - Não envolvido & 1399 & $1.66(0.62)$ & \\
\hline \multirow{4}{*}{ Sensibilidade } & 1 - Vítima & 238 & $2.24(0.87)$ & \multirow{4}{*}{$\begin{array}{l}1>2 \\
1>4 \\
3>2 \\
3>4\end{array}$} \\
\hline & 2 - Agressor & 154 & $1.72(0.61)$ & \\
\hline & 3 - Vítima \& Agressor & 185 & $2.24(0.82)$ & \\
\hline & 4 - Não envolvido & 1399 & $1.63(0.69)$ & \\
\hline
\end{tabular}




\section{Efeito dos estilos parentais no comportamento de bullying: papel mediador da sintomatologia psicopatológica}

De forma a analisar o papel mediador da sintomatologia psicopatológica, foram tidas em conta as relações entre os estilos parentais do pai e da mãe (democrático, autoritário e permissivo) como preditores, quer da sintomatologia psicopatológica (ansiedade, depressão e sensibilidade interpessoal) quer dos comportamentos de bullying (vitimização, agressão, observador). Através da análise de modelos de equações estruturais, foi possível observar um efeito negativo do estilo parental democrático do pai no desenvolvimento de comportamentos de vitimização $(\beta=-.11)$ e agressão $(\beta=-.08)$. Foi possível verificar que o estilo parental autoritário do pai tem um efeito positivo no desenvolvimento de comportamento de vitimização $(\beta=.31)$, agressão $(\beta=.30)$ e não envolvidos $(\beta=.25)$. 0 estilo permissivo no pai apresenta efeito positivo apenas no desenvolvimento de comportamentos de agressão $(\beta=.05)$. De igual modo, foi possível verificar que o estilo democrático no pai prediz negativamente a sintomatologia psicopatológica $(\beta=-.23)$ e o estilo autoritário prediz positivamente a sintomatologia psicopatológica $(\beta=.21)$, assim como se verifica um efeito positivo da sintomatologia psicopatológica no desenvolvimento de comportamentos de vitimização $(\beta=.41)$, agressão $(\beta=.17)$ e não envolvidos $(\beta=.20)$.

Todavia, aquando da testagem do efeito mediador da sintomatologia psicopatológica, verificou-se que o efeito direto inicial do estilo parental democrático do pai com o comportamento vitimização $(\beta=$ .11) perdeu significância $(\beta=-.03)$, verificando-se uma mediação total da sintomatologia psicopatológica ( $\mathrm{SE}=.01, p<.001, \beta=-.093$, IC [-.11; -.07]). Por outro lado, atestou-se que o efeito direto do estilo parental democrático com os comportamentos de agressão $(\beta=-.08)$ perdeu magnitude $(\beta=-.06)$, verificando-se uma mediação parcial (SE $=.00, p=.004, \beta=-.040$, IC [-.05; -.03]), existindo uma predição indireta de $39.6 \%$ através do mediador. Verifica-se que um efeito direto inicial do estilo parental autoritário do pai sobre os comportamentos de vitimização $(\beta=.31)$ diminui $(\beta=.11)$, observando-se uma mediação parcial, (SE $=.01, p<.001, \beta=.068$, IC [.05; .09]) com uma predição indireta de $38 \%$ através do mediador. Verifica-se, igualmente, que o efeito direto do estilo parental autoritário com os comportamentos de agressão $(\beta=.30)$ diminui $(\beta=.08)$, verificando-se uma mediação parcial (SE $=.01, p=.010, \beta=.029$, IC [.02; .04]) com uma predição indireta de $27.1 \%$ através do mediador. Por último, atestou-se que o efeito inicial direto dos comportamentos de não envolvidos $(\beta=.25)$ perdeu magnitude $(\beta=.11)$, observando-se uma mediação parcial (SE $=.02, p=.001, \beta=.034,[.02 ; .05]$ ) com uma predição indireta de $24.1 \%$ através do mediador. No estilo parental permissivo no pai não existe efeito de mediação da sintomatologia psicopatológica sobre os comportamentos de bullying. 0 modelo final (Figura 1) apresenta índices de ajustamento adequados: $\chi^{2}(42)=221.2, \chi^{2} / g l=5.27, p<.001, \mathrm{CFI}=.98, \mathrm{RMR}=.02$, RMSEA $=.05$.

No que concerne à mãe, através da análise de equações estruturais foi possível observar um efeito negativo do estilo parental democrático da mãe no desenvolvimento de comportamentos de vitimização $(\beta=-.08)$ e agressão $(\beta=-.09)$. Foi possível verificar que o estilo parental autoritário da mãe tem um efeito positivo no desenvolvimento de comportamento de vitimização $(\beta=.21)$, agressão $(\beta=.18)$ e observação $(\beta=$.17). 0 estilo permissivo na mãe apenas apresenta efeito positivo no desenvolvimento de comportamentos de agressão $(\beta=.05)$. De igual modo, foi possível verificar que o estilo democrático na mãe prediz negativamente a sintomatologia psicopatológica $(\beta=-.21)$ e o estilo autoritário prediz positivamente a sintomatologia psicopatológica $(\beta=.14)$, assim como se verifica um efeito positivo da sintomatologia psicopatológica no desenvolvimento de comportamentos de vitimização $(\beta=.41)$, agressão $(\beta=.17)$ e observação $(\beta=.21)$.

Todavia, aquando da testagem do efeito mediador da sintomatologia psicopatológica verificou-se que o efeito direto inicial do estilo parental democrático da mãe no comportamento de vitimização $(\beta=$ .08) perdeu significância $(\beta=-.01)$, verificando-se uma mediação total da sintomatologia psicopatológica ( $\mathrm{SE}=.01, p<.001, \beta=-.084$, IC [-.10; -.06]). Por outro lado, atestou-se que o efeito direto do estilo parental democrático com o comportamento de agressão $(\beta=-.09)$ perdeu magnitude $(\beta=-.08)$, verificando-se uma mediação parcial (SE $=.00, p<.001, \beta=-.036$, IC [-.05; -.03]), existindo uma predição indireta de $31.6 \%$ através do mediador. Verifica-se um efeito direto inicial do estilo parental autoritário da mãe sobre os comportamentos de vitimização $(\beta=.21)$ diminui $(\beta=.10)$, observando-se uma mediação parcial ( $\mathrm{SE}=.01, p<.001, \beta=.045$, IC $[.03 ; .06]$ ), com uma predição indireta de $30.4 \%$ através do mediador. Verifica-se igualmente que o efeito direto do estilo parental autoritário com os comportamentos de agressão $(\beta=.18)$ diminui $(\beta=.07)$, verificando-se uma mediação parcial (SE $=.01, p=.022, \beta=.045$, IC $[.01 ; .03])$ com uma predição indireta de $21.3 \%$ através do mediador. Por último, atestou-se que o efeito inicial direto dos comportamentos de não envolvido $(\beta=.17)$ perde magnitude $(\beta=.08)$, observando-se uma mediação parcial (SE $=.02, p=.003, \beta=.023$, IC [.01; .03]) com uma predição indireta de $23 \%$ através do mediador. No estilo parental permissivo na mãe não existe efeito de mediação da sintomatologia psicopatológica sobre os comportamentos de bullying. 0 modelo final (Figura 2) apresenta índices de ajustamento adequados $\chi^{2}(42)=230.1, \chi^{2} / g l=5.48, p<.001, \mathrm{CFI}=.98, \mathrm{RMR}=.02$, RMSEA $=.05$. 


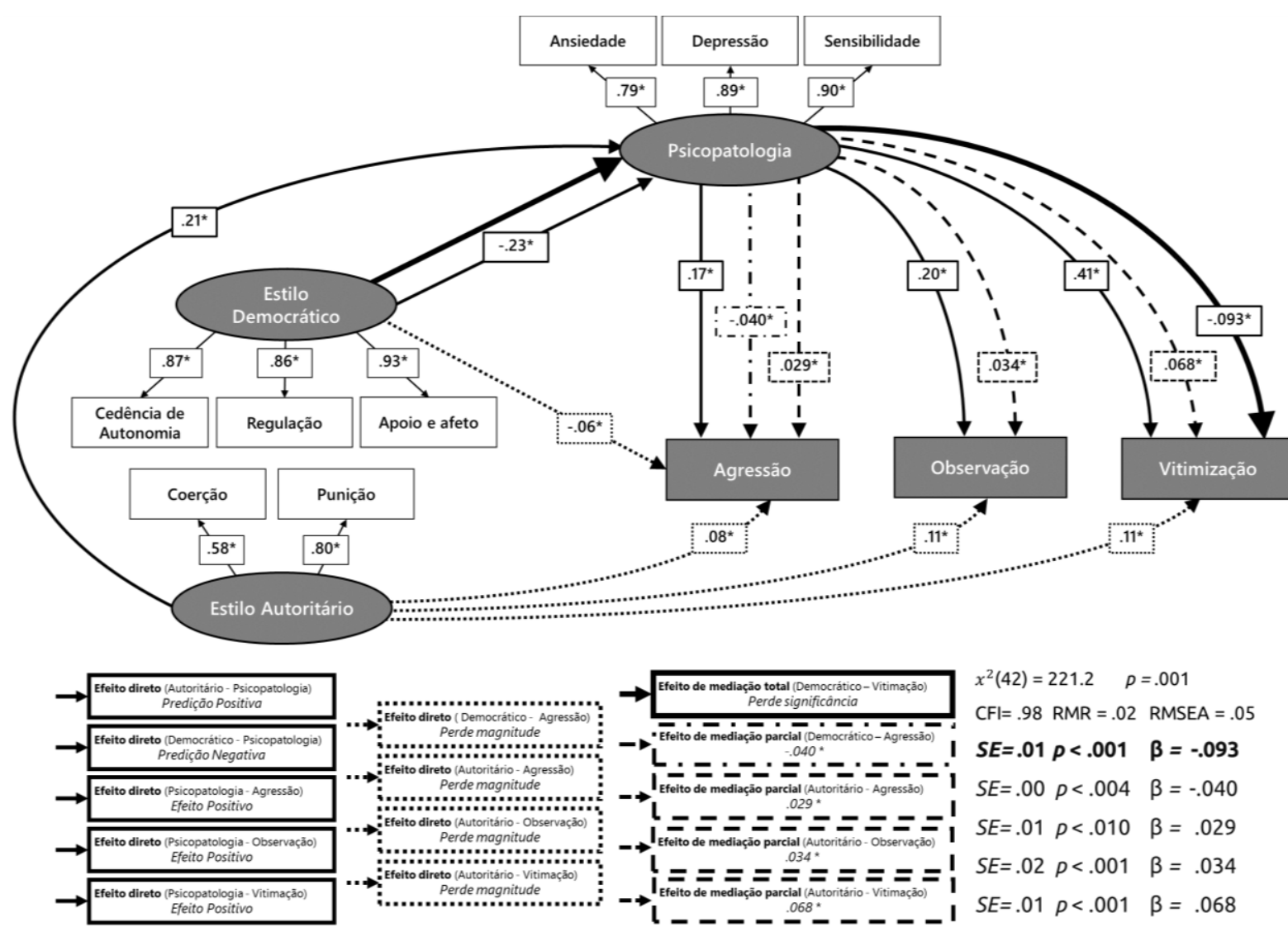

Figura 1. Modelo representativo de efeito mediador da sintomatologia psicopatológica na associação entre estilo parental democrático e autoritário do pai e comportamentos de bullying.

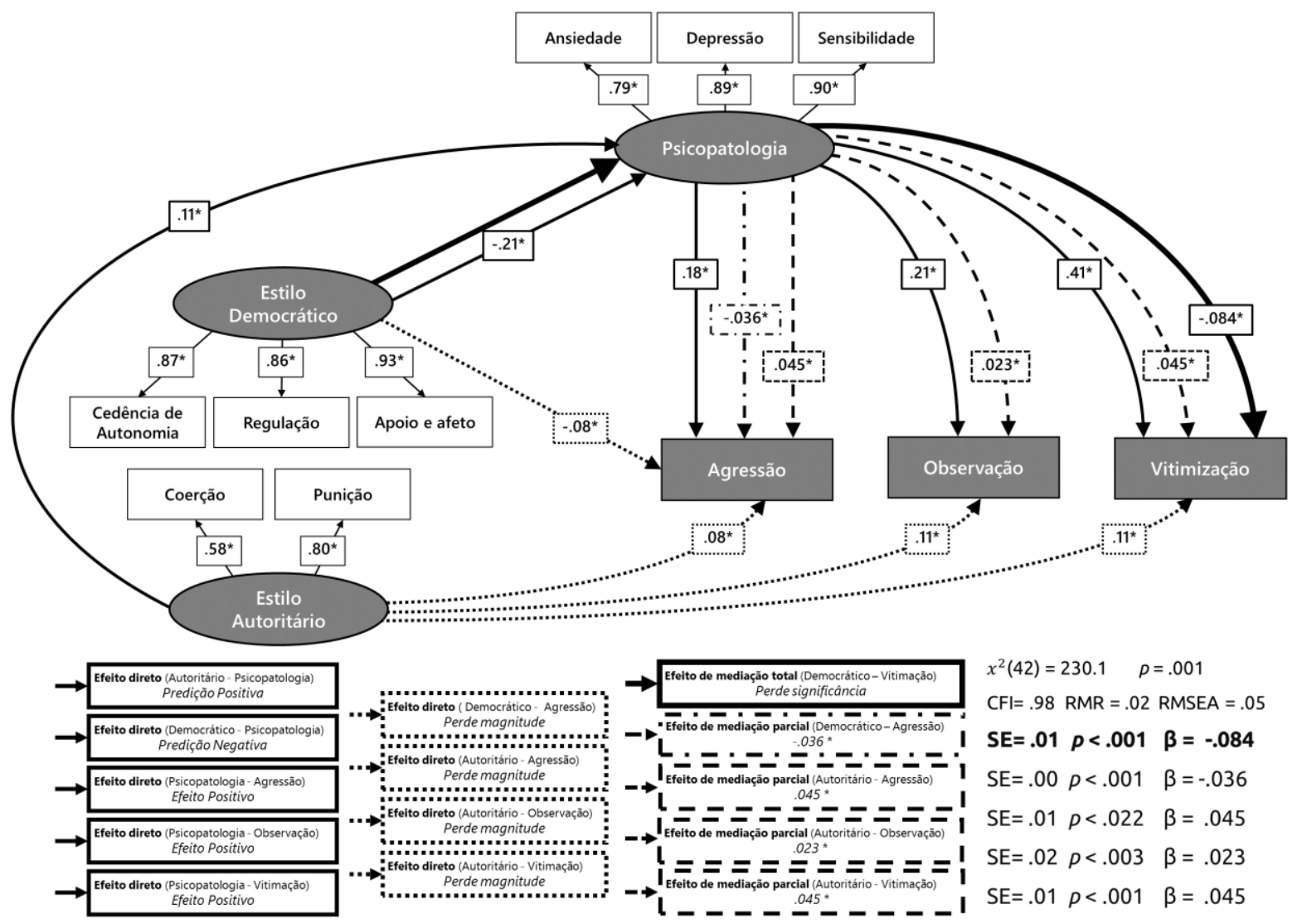

Figura 2. Modelo representativo de efeito mediador da sintomatologia psicopatológica na associação entre estilo parental democrático e autoritário da mãe e comportamentos de bullying. 


\section{DISCUSSÃO}

0 presente estudo teve como principal objetivo analisar o papel dos estilos parentais no desenvolvimento de comportamentos de bullying em adolescentes e jovens adultos, assim como testar o efeito mediador da sintomatologia psicopatológica na associação anterior.

Os resultados apontam para a existência de diferença dos estilos parentais na mãe e no pai face às tipologias de comportamento de bullying. Nesta medida, no estilo parental democrático para a mãe e para o pai, são os sujeitos com tipologia de vítima / agressor que percecionam um estilo mais democrático, comparativamente aos sujeitos não envolvidos. No estilo parental autoritário, são os indivíduos agressores e os indivíduos vítima / agressor que denotam um estilo parental mais autoritário por parte de ambas as figuras parentais, comparativamente aos indivíduos com tipologia de vítima e aos não envolvidos. Por último, verificou-se que no estilo parental permissivo, os agressores são os que percecionam um estilo mais permissivo, quando comparados com os indivíduos vítima / agressor e os não envolvidos. Através do exposto, foi possível verificar que existem menos comportamentos de bullying quando os jovens crescem num estilo parental democrático, pelo que uma educação neste contexto, com níveis de afetividade e controlo equilibrados, parece ser propícia a um desenvolvimento mais adaptativo dos adolescentes e jovens adultos. Por outro lado, os estilos parentais autoritário e permissivo são os que mais propiciam a prática de comportamentos de bullying por parte dos adolescentes e jovens adultos. Nesta medida, pais que mantêm uma relação conflituosa, e são caracterizados por manterem uma distância emocional, tendem a ter papel negativo no desenvolvimento do comportamento futuro dos filhos (Powell \& Ladd, 2010). Segundo a literatura, um estilo parental pautado por compreensão e afeto tende a diminuir comportamentos agressivos (Soares, 2007). Contrariamente, pais que demonstram pouca afetividade, assumem medidas punitivas com os filhos, e impõem regras muito rígidas potenciando comportamentos de agressão e vitimização (Ahmed \& Braithwaite, 2004).

No que diz respeito à variância da sintomatologia psicopatológica face às tipologias de bullying, constatam-se também diferenças significativas. Assim, tal como esperado, jovens com uma tipologia de vítima e vítima / agressor apresentam mais sintomas de ansiedade, depressão e sensibilidade interpessoal comparativamente com os indivíduos com tipologia de agressores e os indivíduos não envolvidos. Indivíduos com tipologia de vítima tendem a ser introvertidos, com baixa autoestima, o que os torna mais suscetíveis a serem submissos. São caracterizados por não serem muito sociáveis, levando a que tenham mais probabilidade de serem vítimas (Olweus, 1993; Ok \& Aslan, 2010; Pontzer, 2010). Os indivíduos de tipo vítima / agressor são em simultâneo vítimas e agressores, podendo mostrar-se menos capazes de estabelecer vínculos afetivos com os pares (Atik \& Güneri 2013; Berger, 2007), replicando comportamentos de agressão similares aos que lhe são proferidos. São indivíduos com níveis significativos de insegurança pessoal, caracterizados como ambivalentes, oscilando entre comportamentos de poder e retração perante os outros (Kaltiala-Heino, Rimpela, Rantanen, \& Rimpela, 2000). Por outro lado, os indivíduos com tipologia de agressores são vistos como tendo uma elevada necessidade de integração no grupo social, em alguns casos são também sujeitos inseguros, apelativos, denotando algum egocentrismo e procura de poder (Gini, 2006). Neste sentido, verificou-se que o grupo dos indivíduos não envolvidos em comportamentos de bullying são os que apresentam menores níveis de sintomatologia psicopatológica, podendo perceber-se mais estabilidade emocional e evitamento da vivência afetiva baseada na dinâmica de agressividade para com o contexto. Assim, segundo a literatura, os indivíduos com tipologia de vítimas, agressores, e vítima / agressores são aqueles que, ainda que exteriorizando de forma diferente, podem apresentar mais necessidade de atenção por parte dos pares e se encontram emocionalmente mais instáveis (Carvalhosa, Lima, \& Matos, 2001; Martins, 2009; Seixas, 2005). Em especial, os sujeitos com tipologia de agressores e vítima/agressores quando não veem correspondidas as suas expectativas de realização, poderão apresentar maior propensão para desenvolver sintomas de depressão, ansiedade e ideias suicidas, quando comparados com indivíduos não envolvidos em comportamentos de bullying (Carvalhosa et al. 2001; Costello, Copeland \& Angold, 2011; Martins, 2005, 2009; Pereira, 2008; Seixas, 2005).

Por último, e de acordo com os objetivos propostos, os resultados apontam para o efeito dos estilos parentais no desenvolvimento de comportamentos de bullying em adolescentes e jovens adultos. Observou-se, para ambas as figuras parentais, um efeito negativo do estilo parental democrático sobre os comportamentos de vitimização e agressão. Por outro lado, verificou-se um efeito positivo do estilo parental autoritário sobre os comportamentos de vitimização, agressão e não envolvimento, e um efeito positivo do estilo parental permissivo no desenvolvimento de comportamento de agressão. Através dos resultados expostos, foi possível verificar que o estilo parental democrático em ambas as figuras parentais, não parece ter efeito nos comportamentos de vítima e agressão. Assim, face à existência de regras e limites equilibrados no seio da relação com os pais, tal como laços emocionais de segurança entre pais e filhos, a ligação a comportamentos de bullying tendem a diminuir. Por outro lado, o estilo parental 
autoritário em ambas as figuras parentais, parece evidenciar um efeito nos comportamentos de vitimização, agressão e observação dos adolescentes. Desta forma, o estilo parental autoritário sugere baixos níveis de afetividade, recorrendo a castigos e punição, pela falta de comunicação, existindo alta exigência e autoridade. Esta relação poderá associar-se ao desenvolvimento de comportamentos pautados por revolta e hostilidade nos adolescentes quer para si próprios, quer com os pares, recriando pouca sensibilidade e atenção para com o sofrimento dos demais. A literatura corrobora esta ideia, na medida em que o estilo democrático, potenciando o acolhimento, sensibilidade e a autonomia emocional dos adolescentes e jovens adultos é frequentemente associado ao não envolvimento em comportamentos agressivos (e.g. Hokoda et al. 2006), ao invés do estilo parental autoritário e o permissivo que assumem contornos de desligamento e menor presença afetiva, potenciando mais insegurança e comportamentos de vitimização e agressão (e.g. Georgiou, 2008; Powell \& Ladd, 2010).

Por último, verificou-se que o estilo parental permissivo exerceu um efeito positivo no desenvolvimento de comportamentos de agressão. De ressaltar que este estilo parental é pautado por um baixo nível de limites, regras ou punições, onde a autoridade parental dos pais perante os filhos é quase inexistente. Esta relação poderá levar a que os adolescentes se sintam menos apoiados e inseguros, e sejam conduzidos a tomar decisões menos adaptativas. Esta aparente autonomia e responsabilização sugere ser um fator preditor de comportamentos inadequados com os pares. Assim, quando a educação não se baseia num equilíbrio emocional, e onde as regras e a afetividade entre os membros da família são escassas, parece haver uma maior propensão para comportamentos agressivos face ao contexto, como forma de resolução interna no sentido de obter algum reconhecimento ou valorização pessoal (Cook et al., 2010). Por outro lado, quando os estilos parentais são pautados pela compreensão e afetividade, podem funcionar como fatores protetores, levando a que os adolescentes se envolvam menos em comportamentos de bullying (e.g. Efobi \& Nwokolo, 2014; Georgiou, 2008).

No presente estudo, destaca-se ainda o papel da sintomatologia psicopatológica, enquanto variável mediadora entre os estilos parentais e o comportamento de bullying. Tal como seria esperado, verificou-se que o estilo parental democrático se associa negativamente com a sintomatologia psicopatológica, nomeadamente a ansiedade, depressão e sensibilidade interpessoal, o que por sua vez conduz a uma ligação negativa com o comportamento de agressão. Assim, aquando da testagem do efeito mediador da sintomatologia psicopatológica, verificou-se que o estilo parental democrático, sendo o mais equilibrado ao nível de regras e limites, não mantém a ligação com o comportamento de vitimização. Todavia, mantém a ligação negativa com a agressão, embora perca magnitude de efeito. Neste sentido, segundo Georgiou e Fanti (2010) e Ok e Aslan (2010), o estilo parental democrático tende a diminuir a ocorrência de comportamentos de agressão e vitimização, no entanto a menor incidência de sintomatologia psicopatológica funciona como um fator protetor para o desenvolvimento dos comportamentos de bullying (Reitman, Rhode, Hupp, \& Altobello, 2002).

Os resultados apontaram, ainda, para que o estilo parental autoritário se associe positivamente com a sintomatologia psicopatológica, predizendo também positivamente os comportamentos de vitimização, agressão e não envolvimento. De destacar que a sintomatologia psicopatológica exerce um efeito mediador parcial nesta relação. A literatura suporta estes dados na medida em que o bullying, exercido de forma contínua, pode provocar baixa autoestima, stresse, ansiedade, baixo rendimento escolar e o despoletar de sintomatologia depressiva e ansiosa, potenciando o desenvolvimento de comportamentos igualmente desadaptativos com os pares (Albino \& Terêncio, 2012; Dracic, 2009). Mais uma vez os resultados apresentados vão ao encontro da literatura, na medida em que adolescentes cujos pais praticam um estilo de educação autoritário, tendencialmente internalizam mais, manifestando dificuldades em fazer face às dificuldades o que pode conduzir ao maior envolvimento em comportamentos apelativos (Reppold \& Hutz 2003; Wolfradt, et al., 2003). Por último, cabe destacar que, ao contrário do esperado, na presente amostra o estilo parental permissivo não denotou um efeito significativo nos comportamentos de bullying, não se verificando um efeito mediador da sintomatologia psicopatológica. Embora reportado na literatura enquanto estilo parental desadaptativo (Simões, et al., 2015), na presente amostra o estilo parental permissivo poderá traduzir uma vivência de jovens mais inseguros com características de afastamento e introversão face aos conflitos, podendo por isso estar menos relacionado com comportamentos de bullying. Os dados obtidos são semelhantes para ambas as figuras parentais, o que sugere, na presente amostra, uma convergência relativamente ao estilo educativo dos pais.

\section{Implicações práticas, limitações e pistas futuras}

0 presente estudo pretendeu contribuir para uma reflexão sobre a temática do bullying, sendo uma realidade cada vez mais presente na sociedade. Este estudo permitiu realçar o papel dos estilos parentais para o desenvolvimento do adolescente e jovem adulto e repercussões na saúde mental e 
comportamentos futuros. Pretende-se que os resultados obtidos possam contribuir para uma maior consciencialização quanto à importância dos estilos parentais e das relações emocionais no seio da família face ao desenvolvimento psicoafetivo do adolescente e jovem adulto.

De destacar algumas limitações no presente estudo, nomeadamente o ambiente onde foi realizada a recolha de dados, pelo que o contexto de sala de aula poderá condicionar as respostas de acordo com o socialmente espectável. Uma outra limitação poderá associar-se à extensão do protocolo e a ampla faixa etária de adolescentes e jovens adultos do ensino secundário e universitário, e o cariz transversal da recolha da amostra limitando a inferência de causalidade entre as variáveis. Destaca-se ainda, embora concordante com a versão original do autor, uma baixa consistência interna das dimensões inerentes ao estilo parental autoritário e permissivo, o que fragiliza a fiabilidade do uso do instrumento.

Apesar das limitações, cabe ressaltar que o presente estudo pretendeu acrescentar à literatura dados relevantes para a maior compreensão dos comportamentos de bullying em adolescentes e jovens adultos. Assim, as conclusões, apesar de suportadas pela literatura, constituem um ponto de partida para o desenvolvimento de estudos longitudinais, com recolhas ao longo de vários períodos desenvolvimentais dos jovens, a fim de validar os resultados apresentados. Futuramente seria oportuno testar o efeito de outras variáveis de relevo, como o contexto cultural e económico dos jovens, assim como fatores de personalidade e os seus efeitos no desenvolvimento de comportamentos agressivos em diferentes etapas ao longo do desenvolvimento. Em próximos estudos, sugere-se uma análise multinível e multinformante, emparelhando ambas as figuras parentais e os jovens num modelo único.

\section{REFERÊNCIAS}

Ahmed, E., \& Braithwaite, V. (2004). Bullying and victimization: cause for concern for both families and $\begin{array}{llllll}\text { schools. Social } & \text { Psychology } & \text { Education, } & 7, & 35-54 .\end{array}$ http://dx.doi.org/10.1023/B:SPOE.0000010668.43236.60

Albino, P., \& Terêncio, M. (2012). Considerações críticas sobre o fenómeno de bullying do conceito ao combate e à prevenção. Revista Eletrónica do CEAF, 1(2), 1-21.

Arnett, J. J. (2006). Emerging adulthood in Europe: A response to Bynner. Journal of Youth Studies, 9, 111123. http://dx.doi.org/10.1080/13676260500523671

Atik G., \& Güneri O. (2013). Bullying and victimization: Predictive role of individual, parental and academic factors. School Psychology International, 34, 658-673. http://dx.doi.org/10.1177/0143034313479699

Baumrind, D. (1966). Effects of authoritative parental control of child behavior. Development, 37, 887907. http://dx.doi.org/10.2307/1126611

Baumrind, D. (1991). The influence of parenting style on adolescent competence and substance use. Journal of Early Adolescence, 11, 56-95. https://doi.org/10.1177/0272431691111004

Baumrind, D. (1997). Necessary distinctions. Psychological Inquiry, 8, 176-229. https://doi.org/10.1207/s15327965pli0803_2

Berger, K. (2007). Update on bullying at school: Science forgotten? Developmental Review, 27, 91-92. http://dx.doi.org/10.1016/j.dr.2006.08.002

Bowlby, J. (1969). Attachment and loss. Vol. 1: Attachment. New York, NY: Basic Books.

Bowlby, J. (1973). Attachment and loss. Vol.2: Separation, anxiety and anger. New York, NY: Basic Books.

Bowlby, J. (1980). Attachment and loss. Vol.3: Loss, sadness and depression. New York, NY: Basic Books.

Brand, S., Gerber, M., Beck, J., Kalak, N., Hatzinger, M., Pühse, U., \& Holsboer-Trachsler, E. (2011). Perceived parenting styles differ between genders but not between elite athletes and controls. Adolescent Health, Medicine and Therapeutics, 2, 9-14. http://dx.doi.org/10.2147/AHMT.S16992

Bynner, J. (2005). Rethinking the youth phase of the life-course: The case for emerging adulthood? Journal of Youth Studies, 8, 367-384. http://dx.doi.org/10.1080/13676260500431628

Byrne, B. M. (2006). Structural equation modelling with EQS: Basic concepts, applications, and programming. $2^{\text {nd }}$ Edition. New Jersey, NJ: Lawrence Erlbaum Associates.

Canavarro, M. (1999). Inventário de sintomas psicopatológicos - BSI. In M. Gonçalves, L. Almeida, \& M. Simões (Eds.), Testes e provas psicológicas em Portugal, vol. III (pp 77-100). Braga: APPORT.

Carvalhosa, S. (2010). Prevenção da violência e do bullying em contexto escolar. Lisboa. Climepsi Editores.

Carvalhosa, S., Lima, L., \& Matos, M. (2001). Bullying - A provocação/vitimação entre pares no contexto escolar português. Análise Psicológica, 4, 523-537. https://doi.org/10.14417/ap.384

Costello, J., Copeland, W., \& Angold, A. (2011). Trends in psychopathology across the adolescent years: What changes when children become adolescents, and when adolescents become adults? Journal of Child Psychology and Psychiatry, 52, 1015-1025. http://dx.doi.org/10.1111/j.14697610.2011.02446.x 
Cook, C., Williams, K., Guerra, N., Kim, T., \& Sadek, S. (2010). Predictors of bullying and victimization in childhood and adolescence: A meta-analytic investigation. School Psychology Quarterly, 25, 65-83. http://dx.doi.org/10.1037/a0020149

Diaz-Aguado, M. J., Arias, R., \& Seoane, G. (2004). Prevención de la violencia y lucha contra la exclusión desde la adolescencia.Vol.1. La violencia entre iguales en la escuela y en el ocio. Estudios comparativos e instrumentos de evaluación. Madrid: Instituto de la Juventud.

Derogatis, L. R. (1993). Brief Symptoms Inventory (BSI): Administration, scoring and procedures manual (3rd Ed.). Minneapolis. MN: National Computers Systems.

Dracic, S. (2009). Bullying and peer victimization. Materia Sócio Medica, 21, 216-219.

Dogde, K., \& Petit, G. (2003). A biopsychosocial model of the development of chronic conduct problems in adolescence. Developmental Psychology, 39, 349-371. http://dx.doi.org/10.1037/00121649.39.2.349

Efobi, A., \& Nwokolo, C. (2014). Relationship between parenting styles and tendency to bullying behaviour among adolescents. Journal of Education \& Human Development, 3, 507-521. http://dx.doi.org/10.5964/ejcop.v2i1.2

Erikson, E. (1968). Identity: Youth and crisis. New York, NY: Norton.

Fan, X., Thompson, B., \& Wang, L. (1999). Effects of sample size, estimation method, and model specification on structural equation modelling fit indexes. Structural Equation Modelling, 6, 56-83. http://dx.doi.org/10.1080/10705519909540119

Field, A. (2005). Discovering statistics using SPSS. London: Sage Publications.

Georgiou, S. (2008). Bullying and victimization at school: The role of mothers. British Journal of Educational Psychology, 78, 109-125. http://dx.doi.org/10.1348/000709907X204363

Georgiou, S., \& Fanti, K. (2010). A transactional model of bullying and victimization. Social Psychology of Education, 13, 295-311. http://dx.doi.org/10.1007/s11218-010-9116-0

Georgiou, S., \& Stavrinides, P. (2013). Parenting at home and bullying at school. Social Psychology of Education, 1-15. http://dx.doi.org/10.1007/s11218-012-9209-z

Gini, G. (2006). Bullying as a social process: The role of group membership in students'

perception of inter-group aggression at school. Journal of School Psychology, 44, 51-65. http://dx.doi.org/10.1016/j.jsp.2005.12.002

Kaltiala-Heino, R., Rimpela, M., Rantanen, P., \& Rimpela, A. (2000). Bullying at school - an indicator of adolescents at risk for mental disorders. Journal of Adolescence, 23, 661-674. http://dx.doi.org/10.1006/jado.2000.0351

Klomek, A., Marrocco, F., Kleinman, M., Schonfeld, I., \& Gould, M. (2007). Bullying, depression, and suicidality in adolescents. American Academy of Child and Adolescent Psychiatry, 46, 40-49. http://dx.doi.org/10.1097/01.chi.0000242237.84925.18

Hokoda, A., Lu, H.-H., \& Angeles, M. (2006). School bullying in Taiwanese adolescents. Journal of Emotional Abuse, 6, 70-90. http://dx.doi.org/10.1300/J135v06n04_04

Hutz, C., \& Bardagir, M. (2006). Indecisão profissional, ansiedade e depressão na adolescência: a influência dos estilos parentais. Psico-USF, 11, 65-73. http://dx.doi.org/10.1590/S141382712006000100008

Laboviti, B. (2015). Perceived parenting styles and their impact on depressive symptoms in adolescent 15-18 years old. Journal of Educational and Social Research, 1, 171-176. http://dx.doi.org/10.5901/jesr.2015.v5n1p171

Lipps, G., Lowe, G., Gibson, R., Halliday, S., Morris, A., Clarke, N., \& Wilson, R. (2012). Parenting and depressive symptoms among adolescents in four Caribbean societies. Child and Adolescent Psychiatry and Mental Health, 6, 31. http://dx.doi.org/10.1186/1753-2000-6-31

Macedo, M. (2010). Adolescência e psicanálise. Interseções possíveis. Porto Alegre: Edipucrs.

Machado, S. (2007). Padrões de vinculação aos pais em adolescentes e jovens adultos e adaptação à Universidade. Revista Portuguesa de Pedagogia, 41, 5-28.

Marôco, J. (2014a). Análise de equações estruturais: Fundamentos teóricos, software e aplicações. Lisboa: Sílabo.

Marôco, J. (2014b). Análise estatística com o SPSS Statistics (6 $6^{\underline{a}}$ ed). Lisboa: Sílabo.

Martins, M. (2005). Agressão e vitimação entre adolescentes, em contexto escolar: Um estudo empírico. Análise Psicológica, 4, 401-425. https://doi.org/10.14417/ap.558

Martins, M. (2009). Maus-tratos entre adolescentes na escola. Lisboa: Editorial Novembro.

Martins, M. J. D., \& Castro, F. V. (2010). How is social competence related to aggression and/or victimization in school? International Journal of Developmental and Educational Psychology, 3, 305315. 
Meier, M., Carr, R., Currie, M., \& Neimeyer, A. (2013). Attachment anxiety and avoidance in coping with bereavement: Two studies. Journal of Social and Clinical Psychology, 32, 315-334. http://dx.doi.org/10.1521/jscp.2013.32.3.315

Michiels, D., Grietens, H., Onghena, P., \& Kuppens, S. (2010). Perceptions of maternal and paternal attachment security in middle childhood: Links with positive parental affection and psychosocial adjustment. Early Child Development and Care, 180, 211-225. http://dx.doi.org/10.1080/03004430903415064

Morris, A., Silk, J., Steinberg, L., Myers, S., \& Robinson, L. (2007). The role of the family context in the development of emotion regulation. Social Development, 16(2), 361-388. http://dx.doi.org/10.1111/j.1467-9507.2007.00389.x

Mota, C. P., \& Rocha, M. (2012). Adolescência e jovem adultícia: Crescimento pessoal, separaçãoindividuação e o jogo das relações. Psicologia: Teoria e Pesquisa, 28, 357-366. http://dx.doi.org/10.1590/S0102-37722012000300011

Neto, L (2005). Bullying: Comportamento agressivo entre estudantes. Jornal de Pediatria, 81, 164-172. http://dx.doi.org/0021-7557/05/81-05-Supl/S164

Newman, K., Harrison, L., Dashiff, C., \& Davies, S. (2008). Relationships between parenting styles and risks behaviors in adolescent health: an integrative literature review. Revista Latino-am Enfermagem, 16, 142-150. http://dx.doi.org/10.1590/S0104-11692008000100022

Nishikawa, S., Sundbom, E., \& Hägglöf, B. (2010). Influence of perceived parental rearing on adolescent self-concept and internalizing and externalizing problems in Japan. Journal of Child and Family Studies, 19, 57-66. http://dx.doi.org/10.1007/s10826-009-9281-y

Nunes, F., \& Mota, C. P. (2018). Parenting styles and dimensions questionnaire - adaptação da versão portuguesa de heterorrelato. Revista Colombiana de Psicología, 27, 117-131. http://dx.doi.org/10.15446/ rcp.v27n1.64621

Olivari, M., Wahn, E., Maridaki-Kassotaki, K., Antonopoulou, K., \& Confalonieri, E. (2015). Adolescent perceptions of parenting styles in Sweden, Italy and Greece: An exploratory study. Europe's Journal of Psychology, 11, 244-258. http://dx.doi.org/10.5964/ejop.v11i2.887

Olweus, D. (1991). Bully/victim problems among school children: basic facts and effects of a school based intervention program. In D. Pepler \& K. Rubin (Eds.), The development and treatment of childhood aggression (pp. 411-448). Hillsdale, NJ: Lawrence Erlbaum Associates.

Olweus, D. (1993). Bullying at school: What we know and what we can do. Oxford, UK: Wiley.

Olweus, D., \& Limber, S. (1999). Blueprints for violence prevention: Bullying prevention program (Book Nine). Boulder, CO: C \& M. Press.

Ok, S., \& Aslan, S. (2010). The school bullying and perceived parental style in adolescents. Procedia Social and Behavioral Sciences, 5, 536-540. http://dx.doi.org/10.1016/j.sbspro.2010.07.138

Pereira, B. (2008). Para uma escola sem violência: estudo e prevenção das práticas agressivas entre crianças (2. ${ }^{a}$ Ed.). Lisboa: Fundação Calouste Gulbenkian.

Pontzer, D. (2010). A theoretical test of bullying behavior: Parenting, personality, and the bully/victim relationship. Journal of Family Violence, 25, 259-273. http://dx.doi.org/10.1007/s10896-0099289-5

Powell, M., \& Ladd, L. (2010). Bullying: A review of the literature and implications for family therapists. The American Journal of Family Therapy, 38, 206. http://dx.doi.org/10.1080/01926180902961662

Reitman, D., Rhode. P., Hupp, S., \& Altobello, C. (2002). Development and validation of the parental authority questionnaire-revised. Journal of Psychopathology and Behavioral Assessment, 24, 119127. http://dx.doi.org/10.1023/A:1015344909518

Reppold, T., \& Hutz, S. (2003). Exigência e responsividade parental como preditores de depressão em adolescentes no sul do Brasil. Avaliação Psicológica, 3, 175-184. http://dx.doi.org/10.1590/S141382712006000100008

Robinson, C. C., Mandleco, B., Olsen, S. F., \& Hart, C. H. (1995). Authoritative, authoritarian, and permissive parenting practices: Development of a new measure. Psychological Reports, 77, 819-830. http://dx.doi.org/10.2466/pr0.1995.77.3.819

Scabini, E., \& Manzi, C. (2011). Family processes and identity. In S. J. Schwartz, K. Luyckx \& V. L. Vignoles (Eds.), Handbook of identity theory and research (Vol. 2, pp. 565-584). New York, NY: Springer.

Seixas, S. (2005). Violência escolar: Metodologias de identificação dos alunos agressores e/ou vítimas. Análise Psicológica, 2, 97-110. https://doi.org/10.14417/ap.75

Silva, J., Morgado, J., \& Maroco, J. (2012). The relationship between portuguese adolescent perception of parental styles, social support, and school behavior. Psychology, 3, 513-517. http://dx.doi.org/10.4236/psych.2012.37074 
Simões, S., Ferreira, J. J., Braga, S., \& Vicente, H. (2015). Bullying, vinculação e estilos educativos parentais em adolescentes do 3o ciclo do ensino básico. Revista Portuguesa de Investigação Comportamental e Social, 1, 30-41. http://dx.doi.org/10.7342/ismt.rpics.2015.1.1.8

Skinner, A., Johnson, S., \& Snyder, T. (2005). Six dimensions of parenting: A motivational model. Parenting: Science and Practice, 5, 175-235. http://dx.doi.org/10.1207/s15327922par0502_3

Skrzypiec, G., Slee, P., Murray-Harvey, R., \& Pereira, B. (2011). School bullying by one or more ways: does it matter and how do students cope? Scholl Psychology Internacional, 32, 288-311. http://dx.doi.org/10.1177/0143034311402308

Soares, I. (2007). Relações de vinculação ao longo do desenvolvimento: Teorias e avaliação. Braga: Psiquilibrios.

Wolfradt, U., Hempel, S., \& Miles, J. (2003). Perceived parenting styles, depersonalization, anxiety and coping behavior in adolescents. Personality and Individual Diferences, 34, 521-532. http://dx.doi.org/10.1016/S0191-8869(02)00092-2

Historial do artigo

Recebido $\quad 20 / 10 / 2017$

Aceite $\quad 05 / 04 / 2018$

Publicado $\quad 12 / 2018$ 